\section{Evaluation of a task-shifting strategy involving peer educators in HIV care and treatment clinics in Lusaka, Zambia}

\author{
Lonny J. Born, 1,2 Chibesa Wamulume, \\ Kim A. Neroda, ${ }^{4}$ Nicole Quiterio, ${ }^{5}$ \\ Mark J. Giganti, 1,6 Mary Morris, 1,7 \\ Carolyn Bolton-Moore, ${ }^{1,6}$ Shelagh Baird, ${ }^{1}$ \\ Maggie Sinkamba, ${ }^{1}$ Stephanie M. Topp, ${ }^{1,6}$ \\ Stewart E. Reid ${ }^{1,7}$ \\ ${ }^{1}$ Centre for Infectious Disease Research in \\ Zambia, Lusaka, Zambia; ${ }^{2}$ University of \\ Washington, Seattle, Washington, USA; \\ ${ }^{3}$ Lusaka District Health Management \\ Team, Lusaka, Zambia; ${ }^{4}$ Emory University \\ School of Medicine, Atlanta, Georgia, \\ USA; ${ }^{5}$ Harvard Medical School, Boston, \\ Massachusetts, USA; ${ }^{6}$ University of \\ Alabama at Birmingham, Alabama, USA; \\ ${ }^{7}$ Elizabeth Glaser Pediatric AIDS \\ Foundation, Washington, DC, USA
}

\section{Abstract}

Rapid expansion of antiretroviral therapy (ART) and a shortage of health care workers (HCWs) required the implementation of a peer educator (PE) model as part of a task-shifting strategy in Lusaka District clinics. The purpose of this study was to evaluate patient and staff perceptions regarding whether the PE program: a) relieved the workload on professional HCWs; and b) delivered services of acceptable quality. Qualitative and quantitative data were gathered from five primary care clinics delivering ART in Lusaka, Zambia. Closed surveys were conducted with 148 patients receiving ART, 29 PEs, and 53 HCWs. Data was imported into Microsoft Excel to calculate descriptive statistics. Six focus group discussions and eight key informant (KI) interviews were conducted, recorded, transcribed, and coded to extract relevant data. Survey results demonstrated that 50 of $53(96.1 \%)$ HCWs agreed PEs reduced the amount of counseling duties required of HCWs. HCWs felt that PEs performed as well as HCWs in counseling patients (48 of 53; 90.6\%) and that having PEs conduct counseling enabled clinical staff to see more patients (44 of 53; 83\%). A majority of patients (141 of 148; 95.2\%) agreed or strongly agreed that PEs were knowledgeable about ART, and 89 of $144(61.8 \%)$ expressed a high level of confidence with PEs performing counseling and related tasks. Focus group and KI interviews supported these findings. PEs helped ease the work burden of HCWs and provided effective counseling, education talks, and adherence support to patients in HIV care. Consideration should be given to formalizing their role in the public health sector.

\section{Introduction}

In the past decade, the critical shortage of professional health care workers (HCWs) has been a major challenge to improving health services in sub-Saharan Africa. ${ }^{1}$ The HIV epidemic exacerbated these shortages by increasing the number of individuals requiring treatment and simultaneously depleting the health workforce through HIV/AIDS-related deaths. ${ }^{2}$ In Zambia, the national adult HIV prevalence rate is $14.3 \% .^{3}$ It is estimated that there are currently 1.1 million individuals with HIV/AIDS. ${ }^{4,5}$ In 2007, Zambia was reported to have 7 physicians, 9.2 clinical officers (equivalent to nurse practitioners in the USA, the UK or Canada) and 113 nurses per 100,000 of the population. These figures mask urban-rural disparities $^{2}$ and barely meet the World Health Organization's (WHO) recommended minimum threshold for health workforce coverage. ${ }^{6}$

Rapid expansion of antiretroviral therapy (ART) from $2004^{7}$ meant that by 2010 more than half of the HIV-infected individuals in some parts of Zambia had access to care and treatment. ${ }^{8}$ In the Lusaka district alone, 132,974 patients were enrolled in HIV care and 82,322 were on ART. ${ }^{8}$ While such a rapid scaleup has been a significant achievement, it has simultaneously strained an already over-burdened professional workforce. ${ }^{9-11}$ Despite declining trends in HIV incidence among young people since 1998, HIV/AIDS remains a major threat in Zambia ${ }^{9}$ and enrollment rates into HIV care and treatment continue to be steep. Innovative strategies are required to handle these challenges while ensuring highquality care. The purpose of this study was to evaluate patient and staff perceptions regarding whether the PE program: i) relieved the workload on professional HCWs; and ii) delivered services of acceptable quality.

One strategy for helping alleviate chronic human resource shortages is task shifting. This involves extending the scope of practice of existing cadres of health workers to allow for the rational redistribution of tasks to make better use of human resources and ease bottlenecks in the service delivery system..$^{12}$ In 2004, the WHO published guidelines recommending that nurses and clinical aides be trained to provide HIV primary care. ${ }^{13}$ In 2007, the United Nations Joint Programme on HIV/AIDS and the President's Emergency Plan for AIDS Relief jointly issued guidelines for the implementation of task shifting to alleviate a chronic
Correspondence: Dr. Stewart E. Reid, Centre for Infectious Disease Research in Zambia, P.0. Box 34681 Plot 2374, Counting House Square, Thabo Mbeki Road, Lusaka, Zambia.

Tel. +260.966.747677. E-mail: stewart.reid@cidrz.org

Key words: peer educators, Africa, Zambia, HIV, adherence, health care workers, task shifting, counseling

Acknowledgments: the authors would like to thank the patients and staff of Chipata, Chilenje, George, Kabwata, and Kanyama Clinics. They also thank the interviewers Kalumba Kamanga and Catherine Mwansa for their tireless patience and effort in data collection, and Kalima Katumbo for significant contributions to database design, data entry, quality assurance and control. They also wish to acknowledge the contributions of the CIDRZ Community Team and peer supervisors, particularly Judith Sakeyo, Susan Chirwa, Fresher Maphiri, Lazarous Chanda, Ephraim Banda, and Constance Mudenda.

Contributions: LJB, study implementation and data collection, management and analysis, first draft of the manuscript; CW, study oversight contribution; KAN, NQ, MS, contribution to initial study concept, protocol design, instrument development; MJG, review of the data management, data analysis, and edits to the manuscript; MM, quality assurance, implementation, and critical edits to the manuscript; CBM, SB, contribution to critical edits; SMT, data management, drafting, and critical edits to the manuscript; SER, protocol design, study oversight, drafting, and critical editing of the manuscript.

Conflict of interest: the sponsor of the study had no role in study design, data collection, analysis, interpretation, or in the writing of the report. None of the authors has a major conflict of interest in this study.

Funding: the program described in this manuscript was supported by a multi-country grant to the Elizabeth Glaser Pediatric AIDS Foundation from the US Centers for Disease Control and Prevention (U62/CCU12354).

Received for publication: 11 February 2011 Accepted for publication: 26 0ctober 2011.

This work is licensed under a Creative Commons Attribution NonCommercial 3.0 License (CC BYNC 3.0).

CC Copyright L.J. Born et al., 2012

Licensee PAGEPress, Italy

Journal of Public Health in Africa 2012; 2:e3

doi:10.4081/jphia.2012.e3

shortage in human resources in high HIVprevalence settings. ${ }^{14}$

A number of studies have demonstrated that for HIV-infected individuals enrolled in ART, detailed information on the medication regi- 
men (particularly during the first weeks after initiating ART), and education about side effects and disease progression ${ }^{15,16}$ contribute to improved patient knowledge, stronger commitment to ART adherence, and ultimately more successful patient outcomes. ${ }^{17-20}$ Task shifting these activities to peer educators (PEs) has been identified as a valuable strategy. Recent studies show that PEs have been used to good effect by taking on non-clinical but critical duties in the clinic, freeing up more highly-trained health care professionals to focus on clinical duties. ${ }^{21-28}$ In 2008, Médecins Sans Frontières reported that switching to a lower cadre may provide cost benefits for patients and health systems. ${ }^{29} \mathrm{~A}$ 2010 review of 84 articles examining the effect of task shifting found overwhelmingly positive results, including increased clinical capacity, ART uptake, and improved quality of patient care. $^{30}$

\section{Lusaka peer educator model}

In 2004, the Center for Infectious Disease Research in Zambia (CIDRZ) partnered with the Zambian Ministry of Health (MOH) and the Lusaka District Health Management Team (LDHMT) to initiate a PE program using the WHO-recommended task-shifting guidelines. ${ }^{31}$ The Lusaka program selects PEs from community HIV/AIDS support groups that meet at the clinic. PEs are selected for their literacy skills and are usually HIV-positive and undergoing treatment themselves. Initially, PE responsibilities were limited to supporting professional staff in delivering adherence counseling. Over time, PEs have become an established and integral presence in HIV treatment centers. ${ }^{25,28} \mathrm{PEs}$ are required to have grade 12 level literacy and undergo training in psychosocial counseling, ${ }^{32}$ behavior change, HIV basics, treatment adherence, ${ }^{33}$ and positive living before starting work. PEs are contracted to work four hours daily, five days per week, and are supervised by on-site ART nurses and a CIDRZ peer supervisor. PE duties include: conducting health talks, accessing patient files, filing patients' lab results, enrolling newlydiagnosed HIV-positive patients, assisting with patient flow, medication adherence counseling, and providing psycho-social support. PEs also conduct community sensitization meetings, counseling of couples, and patient tracking visits within their communities. PEs receive a monthly stipend of 600,000 Zambian Kwacha (approximately USD120) from CIDRZ. Since July 2005, more than 157 PEs have been trained and stationed in both urban and rural clinics.

\section{Materials and Methods}

\section{Study setting and site selection}

Zambia's capital, Lusaka, has a population of approximately 1.1 million served by 27 public primary health centers managed by a district-level health office. For this evaluation, five health centers with ART clinics operating for a minimum of three years were purposefully selected. Sites were selected by consensus of key stakeholders and Neighborhood Health Committee (NHC) members to represent clinics serving catchment populations within Lusaka with different geographical and sociodemographic characteristics. The protocol was approved by the Institutional Review Board at the University of Alabama at Birmingham and the Research Ethics Committee of the University of Zambia. Informed consent was obtained from all participants.

\section{Study design}

This study used a mixed-methods approach to evaluate the perceptions of health care workers, patients and peer educators of the role of PEs in reducing the workload of professional HCWs and the acceptability of PEs as members of clinic health care teams. In the first instance, a questionnaire was used to gather information from a sample of each of the three groups. Data from the questionnaire were collated and reviewed, and conflicting or unclear findings subsequently clarified through focus group discussions (FGD) and semi-structured interviews with key informants (KI). Quantitative analysis was carried out with Microsoft Excel 2007 (Microsoft Corp, WA, USA). Descriptive statistics were obtained for tabulated responses to selected questions. Multiple rounds of inductive thematic coding were used to analyze qualitative data from FGD and interview narratives. The evaluation was carried out between 15 December 2008 and 13 February 2009.

\section{Questionnaires (Appendices A, B and C)}

Different questionnaires were administered in private to HCWs, PEs, and patients. The HCW questionnaire (Appendix A) was designed to quantify provider workload and to gauge PE contributions in decreasing $\mathrm{HCW}$ workload. Only HCWs were eligible to participate; clerical staff were excluded. The PE questionnaire (Appendix B) examined the perception of PEs of their contribution towards decreasing HCW workload and improving patient care. All PEs were eligible to participate. The patient questionnaire (Appendix C) collected information regarding patient interaction with all HCWs, including PEs, patient understanding of the PE's role in the clinic, and relative satisfaction across lay and professional providers. Patients had to be over 19 years of age and enrolled in a participating clinic to be eligible. Patients were asked individually by study staff if they wished to participate and were informed of their right to refuse with no adverse consequence to their care. Questionnaires were offered in all three local languages: English, Bemba, or Nyanja.

\section{Focus group discussions (Appendix D)}

Focus group discussions (FGDs) and $\mathrm{KI}$ interviews moderated by two trained Zambian interviewers were held to gain additional indepth information. Two FGDs were conducted with each of the HCWs, PEs and patients, which equals six. FGDs were conducted in a neutral venue. All PEs were eligible to participate. HCWs and patients who completed the questionnaire were invited to participate. FGDs were conducted in the participant's language of preference. Participants were informed that discussions would be recorded and they received financial compensation for their time and transport.

\section{Key informant interviews}

Interviews were conducted with program supervisory staff, NHC representatives, those in charge of the ART clinic, LDHMT coordinators, and medical personnel. KIs were identified by stakeholders or CIDRZ staff during the course of the study. Interviews were held in private.

\section{Results}

\section{Questionnaires}

The 53 HCW participants (Table 1) were 40 nurses (75.5\%), 6 clinical officers (11.3\%), and 7 pharmacy technicians (13.2\%). A majority (50 of $53 ; 96.1 \%$ ) agreed that PEs reduced the amount of counseling required of clinical staff and that PEs did as good a job of counseling patients as professional HCWs (48 of 53; $90.6 \%$ ). Most HCWs (44 of 53; 83\%) felt they could attend to more patients because PEs performed adherence counseling. However, 30 of 53 HCWs (57\%) did not feel that PEs' assistance with adherence counseling enabled them to spend more time with patients (Table 2).

Twenty-nine PEs answered the questionnaire (Table 1). Of these, 28 of 29 (96.6\%) believed they reduce the amount of counseling professional HCWs must perform; 23 of 29 (79.3\%) believed they do as good a job at counseling as professional HCWs; 22 of 29 (75.9\%) believed their work enables HCWs to see and spend more time with patients; and 25 of 29 (86.2\%) believed their work enables HCWs to spend more time talking to patients about 
issues other than adherence (Table 2).

A majority of PEs (20 of 29; 69\%) rated their job satisfaction as satisfied or very satisfied, while 4 of 29 (13.7\%) were unsatisfied or very unsatisfied. The top three factors influencing PEs' motivation, performance, and retention were: i) helping other people living with HIV/AIDS, 27 of 29 (93.1\%); ii) learning new skills, 13 of 29 (44.9\%); and iii) being a role model for other people living with HIV/AIDS, 12 of 29 (41.3\%) (Table 3). PEs acknowledged other factors affecting their job satisfaction, including informal professional and personal interactions, stipends, and overwork, but ranked these common themes low overall.

Of the 62 male (41.5\%) and 86 female (58.5\%) patients surveyed (Table 1), 141 (95.2\%) agreed or strongly agreed that PEs were knowledgeable about ARV treatment (Figure 1), 89 (61.8\%) expressed a high level of confidence in PEs and the services they provided (Figure 2), and all (100\%) agreed or strongly agreed that they were comfortable with PEs as adherence counselors. Most patients agreed or strongly agreed that PEs were informed and knowledgeable $(n=141$; 95.2\%) and spent enough time discussing concerns $(\mathrm{n}=135 ; 91.9 \%)$ and explaining things clearly $(n=145 ; 98 \%)$. Over half $(n=90 ; 64.3 \%)$ felt PEs did as good a job at counseling as professional HCWs, while $35.7 \% \quad(n=50)$ of patients disagreed with this statement.

Despite these findings, most patients indicated they had high levels of confidence in clinical officers $(n=136 ; 93.8 \%)$ and pharmacists $(n=121 ; 83.4 \%)$. Some patients expressed a lack of confidence in PEs $(n=3 ; 2.1 \%)$, clinical officers $(\mathrm{n}=2 ; 1.4 \%)$, or pharmacy technicians $(n=1 ; 0.7 \%)$, with slightly more expressing low confidence in nursing staff $(n=7$; 4.9\%) (Figure 2).

\section{Focus group discussions and inter views}

FGDs and KI interviews indicated positive attitudes towards the PE program. Focus group discussions identified the following PE activities and outcomes as being of particular value: - enrollment of new patients;

- assistance with health education activities;

- assistance with follow-up activities;

- provision of adherence counseling;

- improved ability of professional staff to attend to clinical duties due to PEs' assistance with recording vital signs, filing charts, and labeling medicine bottles;

- improved patient awareness of drug adherence, risky behavior, and family involvement.

Interview data supported questionnaire results, confirming that PEs were seen by HCWs as providing quality counseling and adherence support, and contributing significantly to reducing their workload. PEs identi- fied themselves as expert patients, which enabled them to relate to patients and help them navigate the health care system. Both PEs and HCWs identified PEs as role models for patients.

Interview data of PEs revealed a majority are satisfied with their job and find their work rewarding. Common motivators include: compassion for other patients, helping to save lives, a desire to continue educating other patients and to reach out to stigmatized people living with HIV/AIDS (PLWHAs), and encouraging ART adherence.

KIs similarly agreed that PEs were doing a

Table 1. Questionnaire distribution.

\begin{tabular}{|c|c|c|c|}
\hline Demographic & $\begin{array}{l}\text { Patient } \\
(\mathrm{n}=148)\end{array}$ & $\begin{array}{c}\text { Health care } \\
\text { worker }(\mathrm{n}=53)\end{array}$ & $\begin{array}{l}\text { Peer educator } \\
(\mathrm{n}=29)\end{array}$ \\
\hline $\begin{array}{l}\text { Job title } \\
\text { Nurse } \\
\text { Clinical officer } \\
\text { Medical officer } \\
\text { Pharmacy technician }\end{array}$ & NA & $\begin{array}{c}40(75.5 \%) \\
6(11.3 \%) \\
0 \\
7(13.2 \%)\end{array}$ & NA \\
\hline $\begin{array}{c}\text { Gender } \\
\text { Male } \\
\text { Female }\end{array}$ & $\begin{array}{l}62(41.5 \%) \\
86(58.5 \%)\end{array}$ & $\begin{array}{c}6(11.3 \%) \\
47(88.7 \%)\end{array}$ & NA \\
\hline
\end{tabular}

Table 2. Perceptions of peer educators (PE) and health care workers (HCW) of PE adherence counseling contributions.

\begin{tabular}{lll} 
& $\begin{array}{l}\text { Peer educators } \\
(\mathrm{n}=29) \\
\text { Agree or } \\
\text { strongly agree }\end{array}$ & $\begin{array}{l}\text { Health care workers } \\
(\mathrm{n}=53) \\
\text { Agree or } \\
\text { strongly agree }\end{array}$ \\
$\begin{array}{l}\text { PEs help reduce the amount of counseling } \\
\text { the rest of the clinic staff must perform. }\end{array}$ & $28(96.6 \%)$ & $\begin{array}{l}50(96.1 \%), \\
1 \text { missing }\end{array}$ \\
$\begin{array}{l}\text { PEs do as good a job of counseling patients } \\
\text { as health care workers. }\end{array}$ & $23(79.3 \%)$ & $48(90.6 \%)$ \\
\hline $\begin{array}{l}\text { Health staff are able to see more patients. } \\
\begin{array}{l}\text { Nurses, clinical officers, pharmacists are able } \\
\text { to spend more time with individual patients. }\end{array}\end{array}$ & $\begin{array}{l}22(75.9 \%) \\
1 \text { missing }\end{array}$ & $44(83.0 \%)$ \\
$\begin{array}{l}\text { Health staff can spend more time talking to } \\
\text { patients about issues other than adherence. }\end{array}$ & $25(86.2 \%)$ & $30(56.6 \%)$ \\
$\begin{array}{l}\text { HCWs spend less time talking } \\
\text { to patients about adherence. }\end{array}$ & N/A & $45(84.9 \%)$ \\
\hline
\end{tabular}

Table 3. Factors most influencing motivation, performance, and retention of ART peer educators.

\begin{tabular}{lc} 
What are the three factors that most influence your satisfaction & $(\mathbf{n = 2 9 )}$ \\
as an ART peer educator? & 27 \\
Helping other people living with HIV/AIDS through counseling & 13 \\
Learning new skills & 12 \\
\hline Being a role model for other people living with HIV/AIDS & 7 \\
Working relationships with ART clinic staff & 6 \\
\hline Conducting health talks and community sensitization activities & 5 \\
Getting respect from people in my community because of this work & 4 \\
\hline Having opportunities for job advancement & 4 \\
Receiving a stipend & 3 \\
\hline Being of assistance to the clinic support groups & 3 \\
Having a supervisor and getting feedback on how to improve & 1 \\
\hline Having stipend paid on time & 0 \\
The number of patients I must counsel & 3 \\
\hline Other
\end{tabular}


good job of meeting responsibilities, especially in relation to adherence counseling. One key informant stated: You find that people now are given health education about the ARVs. Another commented: Patients now understand what time they have to take medication and are reminded of their next visit. Despite these affirmations, PEs expressed a desire for additional specialized training in counseling couples and disclosure counseling, and roles related to pharmacy and blood drawing.

FGDs and KI interviews highlighted a number of challenges to PE retention. These include lack of or late payment of stipends, death/illness, heavy workload, long hours, and job opportunities elsewhere. Workload was an especially strong theme. FGDs identified that a number of PEs work as many as ten hours daily despite their contract stipulating a maximum of four hours per day.

\section{Discussion}

Zambia has one of the world's largest ART programs with over 350,000 enrolled at the end of 2010. This evaluation demonstrates that there is a strong perception among patients and professional HCWs that PEs play an important role in supporting the provision of these services. Both PEs and HCWs agreed that, as adherence counselors, PEs helped reduce the amount of counseling that other clinic staff had to perform. KI interviews identified an additional range of non-clinical tasks taken on by PEs, seen by HCWs as easing their workload. This study sought to assess not only whether PEs reduced the burden of work on professional HCWs, but also whether PEs were judged by their professional colleagues and patients as being able to deliver quality services ${ }^{15,29}$ and, specifically, whether PEs were perceived as reputable and trustworthy. Results demonstrated a high level of acceptability and professional respect for PEs amongst both HCWs and patients. The latter expressed a higher level of overall confidence in PEs than nurses. Patients similarly expressed high levels of comfort with PEs as adherence counselors, generally noting that PEs were wellinformed and knowledgeable, and spent adequate time discussing concerns and explaining key information clearly. Consistent with findings from other Zambian and sub-Saharan studies, ${ }^{22,25,28}$ these results confirm that task shifting is acceptable as well as feasible.

While findings from this study were generally positive, this evaluation highlights areas for improvement. A third $(\mathrm{n}=52 ; 36 \%)$ of patients reported being only somewhat confident in PEs and $11(8 \%)$ patients felt that PEs did not spend enough time discussing concerns relating to adherence. If we extrapolate this finding across the entire Lusaka district, it is possible that approximately 6500 patients may have had similar concerns. Moreover, 44 (30\%) patients also felt that PEs were not doing as well at counseling as an HCW, suggesting that more rigorous training and supervision will be necessary to improve PEs' capacity to deliver accurate and timely information and counseling. ${ }^{15-}$ 17,20 To date, both initial recruitment and training of Lusaka PEs and subsequent supervision have been provided by CIDRZ with LDHMT oversight. In the future, stakeholders who oversee the PE program will need to consider more regular and thorough training, performance review, and supportive supervision to help improve performance of PEs, and the overall efficacy of HIV care and treatment.

PEs now play a critical role in the provision of HIV care and treatment in the Lusaka urban clinics. They are viewed by both HCWs and patients as key members of the clinic team. However, FGDs revealed that PEs wish to be viewed (particularly by professional staff) as formal members of the health care team. For example, PEs expressed a high level of job satisfaction but also articulated a desire for training in blood drawing and injections (a skill-set clearly associated with professional cadres). We believe that PEs do not necessarily want to change their job description per se but are seeking ways to bolster their status through the acquisition of higher-level skills. These

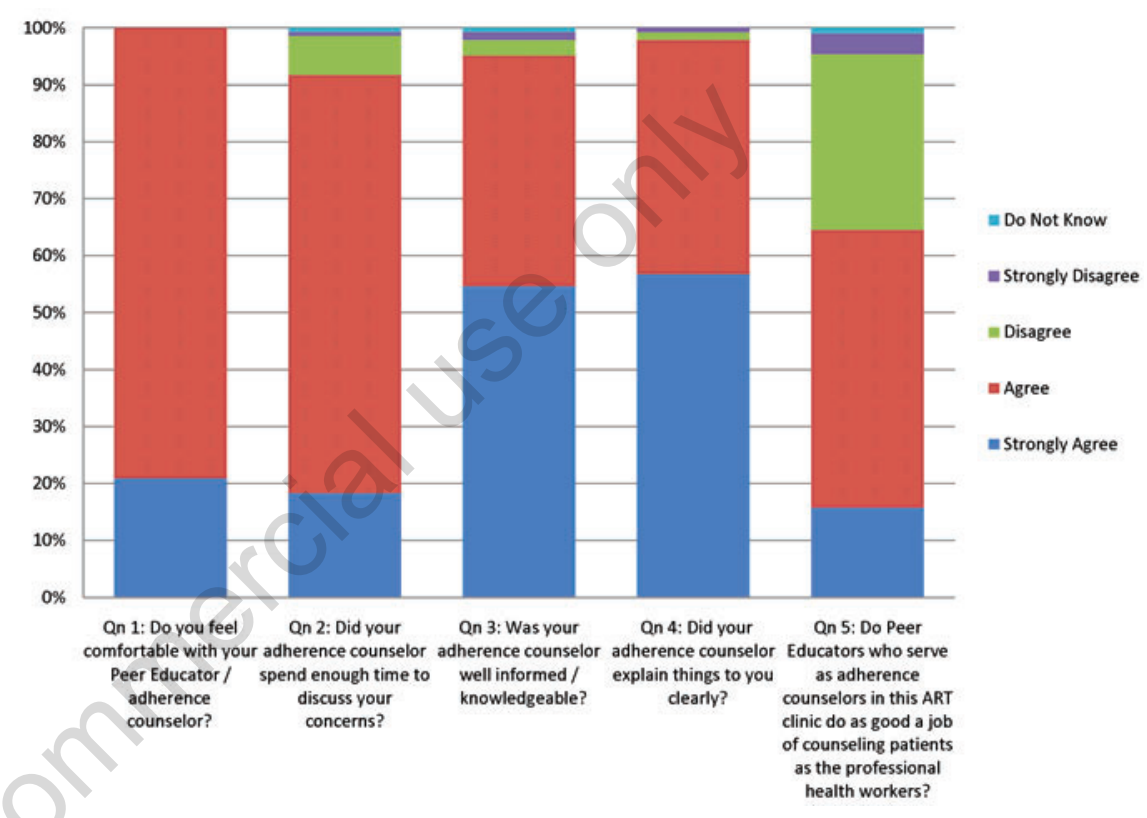

Figure 1. Patient confidence in peer educators as adherence counselors.

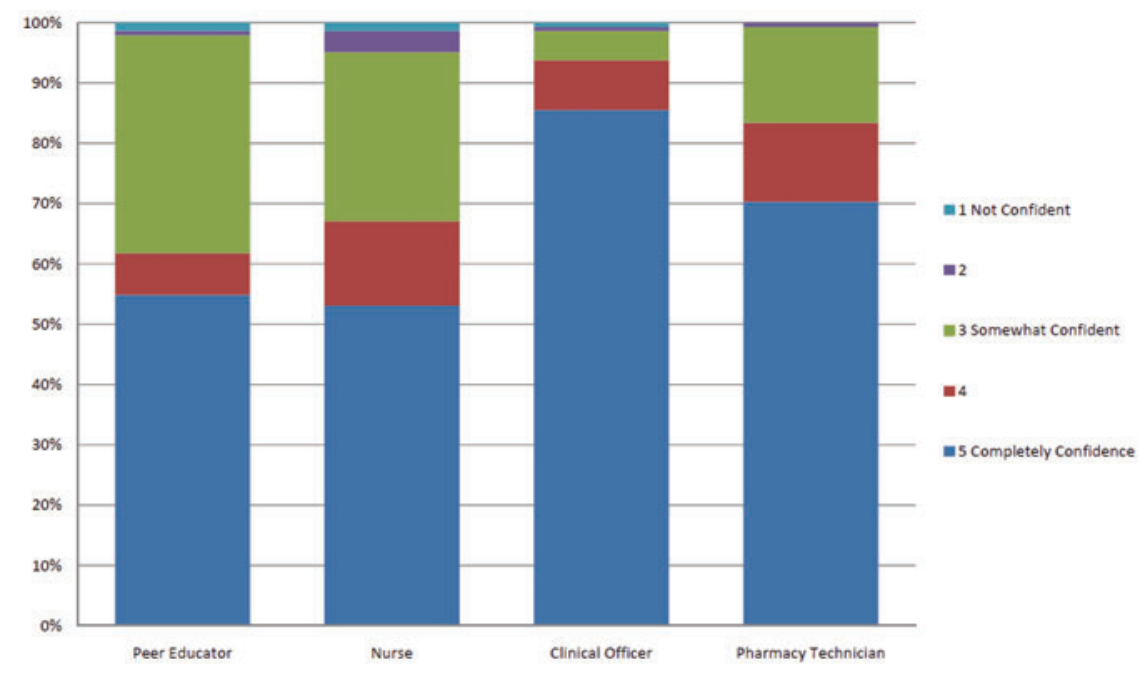

Figure 2. Patient confidence in lay and professional health care workers. 
results suggest PEs are more likely to remain satisfied and productive members of a clinic if in the short-term they are formally recognized as members of the health care team, and in the middle- to long-term are provided with opportunities to acquire new skills and develop professionally. A linked issue is that as stipend volunteers PEs work a substantial number of unpaid hours in order to take on essential nonclinical tasks that existing HCWs do not have time for. Given ongoing shortages within professional cadres and with the burden of HIV care and treatment only set to rise, this study suggests that it would be prudent to investigate further scaling-up of similar task-shifting strategies.

The Zambian $\mathrm{MOH}$ is currently reviewing the voluntary role of PEs with a view to standardizing and formalizing their positions. Understanding how to ensure the long-term sustainability of such a program remains complex and lies beyond the scope of this study. Nonetheless, issues that should be addressed include a need for ongoing supervision and training, and implications for on-site management. ${ }^{34}$ We recommend a certification program with clearly defined scope of practice, competencies and recognition within the national $\mathrm{MOH}$ structure, improved recognition of worker status, associated compensation, and more regulated work hours. In particular, further country-specific research is required to understand the cost-effectiveness and longterm financial implications of formally incorporating a PE program into the public health sector workforce. ${ }^{12,35}$ Until then, responsibility for training and remuneration of PEs will fall squarely on non-government and donor-funded organizations who must continue to work with the public sector to support and research the most effective models for this setting.

\section{Limitations}

This study was conducted in five purposefully selected sites. It may not be appropriate to generalize our findings in other settings. Responses of PEs and HCWs were subject to bias. The patient questionnaire was lengthy and included several technical sections. Both patients and interviewers complained of questionnaire fatigue. Incomplete patient responses from several sections were excluded from the data analysis. We are encouraged, however, by the consistency of our findings with other similar studies.

\section{Conclusions}

This PE program evaluation has demonstrated that utilizing PEs trained specifically to counsel, educate, and provide adherence support to PLWHAs helps ease the work burden for other HCWs in HIV clinics. Patient and HCW confidence levels in the program are high, further supporting the importance of a PE program and its vital role in providing patient care services. Consideration should be given to formalizing the role of PEs in the public health sector. More research needs to be conducted in the form of cost-benefit analyses and economic impact studies to better understand a long-term role for the PE cadre and its sustainability.

\section{References}

1. Chen L, Evans T, Anand S, et al. Human resources for health: overcoming the crisis. Lancet 2004;364:1984-90.

2. Samb B, Celletti F, Holloway J, et al. Rapid expansion of the health workforce in response to the HIV epidemic. N Engl $\mathrm{J}$ Med 2007;357:2510-4.

3. Office CS. Zambian Demographic Health Survey, Lusaka, 2007.

4. UNAIDS. Epidemiological Fact Sheet on HIV and AIDS, 2008, Zambia Country Profile. Geneva: Joint United Nations Programme on HIV/AIDS, 2008.

5. WHO. Zambia Health Profile. Geneva: World Health Organization 2010.

6. WHO. The World Health Report 2006. Geneva: World Health Organization 2006.

7. Stringer JS, Zulu I, Levy J, et al. Rapid scale-up of antiretroviral therapy at primary care sites in Zambia: feasibility and early outcomes. JAMA 2006;296:782-93.

8. Government of the Republic of Zambia, Ministry of Health. National Antiretroviral Program. Ndeke House, Lusaka, Zambia, 2009.

9. CSO. Zambia Demographic and Health Survey 2007. Calverton, Maryland: Central Statistical Office, Ministry of Health, Tropical Diseases Research Centre, University of Zambia, 2009.

10. ILO. HIV/AIDS and work: global estimates, impact and response. Geneva: International Labor Organization, 2004.

11. Torpey KE, Kabaso ME, Mutale LN, et al. Adherence support workers: a way to address human resource constraints in antiretroviral treatment programs in the public health setting in Zambia. PLoS One 2008;3:e2204 18493615.

12. Celletti F, Wright A, Palen J, et al. Can the deployment of community health workers for the delivery of HIV services represent an effective and sustainable response to health workforce shortages? Results of a multicountry study. AIDS 2010;24:S45-57.

13. WHO. Integrated Management of Adolescent and Adult Illness. Geneva: World Health Organization, 2004.

14. WHO. Treat train retain. Task shifting: Global recommendations and guidelines.
Geneva, World Health Organization, 2007.

15. Schneider H, Blaauw D, Gilson L, et al. Health systems and access to antiretroviral drugs for HIV in Southern Africa: service delivery and human resources challenges. Reprod Health Matters 2006;14:1223.

16. Jaffar S, Mbidde E, Robb A, et al. Scale-up of antiretroviral therapy in sub-Saharan Africa: priorities for public health research. Trop Med Int Health 2007; 12:1009-10.

17. Bisson GP, Gross R, Bellamy S, et al. Pharmacy refill adherence compared with CD4 count changes for monitoring HIVinfected adults on antiretroviral therapy. PLoS Med 2008;5:e109.

18. Chi BH, Cantrell RA, Zulu I, et al. Adherence to first-line antiretroviral therapy affects non-virologic outcomes among patients on treatment for more than 12 months in Lusaka, Zambia. Int J Epidemiol 2009;38:746-56.

19. Coetzee D, Boulle A, Hildebrand K, et al. Promoting adherence to antiretroviral therapy: the experience from a primary care setting in Khayelitsha, South Africa. AIDS 2004;18:S27-31.

20. Tuldra A, Wu AW. Interventions to improve adherence to antiretroviral therapy. J Acquir Immune Defic Syndr 2002;31:S1547.

21. Baiden F, Akanlu G, Hodgson A, et al. Using lay counsellors to promote communitybased voluntary counselling and HIV testing in rural northern Ghana: a baseline survey on community acceptance and stigma. J Biosoc Sci 2007;39:721-33.

22. Hermann K, Van Damme W, Pariyo GW, et al. Community health workers for ART in sub-Saharan Africa: learning from experience--capitalizing on new opportunities. Hum Resour Health 2009;7:31.

23. Medley K, Schmid G, Sweat MD. Systematic review of the efficacy of peer education in reducing HIV sexual risk behavior in developing countries. Poster Presentation: International Conference on AIDS, Jul 11-16; Bangkok, Thailand, 1994.

24. PPfCC. A cultural model of mentoring and peer support shows results, Pew Partnership for Civic Change. Nation's Cities Weekly, 1997.

25. Sanjana P, Torpey K, Schwarzwalder A, et al. Task-shifting HIV counselling and testing services in Zambia: the role of lay counsellors. Hum Resour Health 2009; 7:44.

26. Shetty AK, Mhazo M, Moyo S, et al. The feasibility of voluntary counselling and HIV testing for pregnant women using community volunteers in Zimbabwe. Int $\mathbf{J}$ STD AIDS 2005;16:755-9.

27. Welsh M, Lamptey, P, Brokenshire, C, et al. 
Peer education: a critical assessment of an approach to AIDS prevention. Poster Presentation: International Conference on AIDS, June 16-21, 1991.

28. Morris MB, Chapula BT, Chi BH, et al. Use of task-shifting to rapidly scale-up HIV treatment services: experiences from Lusaka, Zambia. BMC Health Serv Res 2009;9:5.

29. Zachariah R, Ford N, Philips M, et al. Task shifting in HIV/AIDS: opportunities, challenges and proposed actions for subSaharan Africa. Trans R Soc Trop Med Hyg 2009;103:549-58.
30. Callaghan M, Ford N, Schneider H. A systematic review of task- shifting for HIV treatment and care in Africa. Hum Resour Health 2010;8:8.

31. WHO. Task shifting: rational redistribution of tasks among health workforce teams, global recommendations and guidelines. Geneva, World Health Organization, 2007.

32. CRS. Training Curriculum: Psychosocial Care and Counseling for HIV Infected Children and Adolescents. Washington DC: Catholic Relief Services \& AIDS Relief, 2008.
33. CIDRZ. Training: HIV Basics, Adherence and Positive Living. Lusaka, Zambia: Centre for Infectious Disease Research in Zambia, 2008.

34. Rowe AK, de Savigny D, Lanata CF, Victora CG. How can we achieve and maintain high-quality performance of health workers in low-resource settings? Lancet 2005; 366:1026-35.

35. Schneider H, Lehmann U. Lay health workers and HIV programmes: implications for health systems. AIDS Care 2010;22:60-7. 
